\title{
On the geometric mean of a pair of oriented, meromorphic foliations, Part I
}

\author{
Joel C. Langer ${ }^{*}$ and David A. Singer
}

*Correspondence: joel.langer@case.edu Department of Mathematics, Applied Mathematics, and Statistics, Case Western Reserve University, Euclid Avenue, Cleveland, $\mathrm{OH}$, USA

\section{Abstract}

The geometric mean of a pair of oriented, meromorphic foliations on a Riemann surface is an unoriented foliation; the quadratic differential defining the latter is the product of the linear differentials defining the former. For instance, geometric means of pairs of oriented circle pencils in the Riemann sphere may be used to represent confocal families of (Euclidean or non-Euclidean) conics.

Keywords: Confocal conics, Meromorphic quadratic differential, Meromorphic foliation, Non-Euclidean geometry

\section{Introduction}

This is Part I of an exposition on geometric mean foliations, with applications to confocal families of (Euclidean and non-Euclidean) conics. Our broader goal is to demonstratefor the benefit of non-specialists - the value of quadratic differentials for geometry and vice versa (continuing a theme of [2-5]). We begin by explaining the central idea and mathematical context of this exposition.

If $u$ and $v$ are a pair of non-zero vectors (or oriented lines) based at a point $p$ in the Euclidean plane, one may define their geometric mean $w=\sqrt{u v}$ to be the unoriented line through $p$ which bisects the angle formed by $u$ and $v$. If $u$ and $v$ are vector fields (or oriented line fields), $w=\sqrt{u v}$ is the unoriented line field defined pointwize as above, except at singularities of $u$ and $v$. Finally, if $\mathcal{F}_{u}$ and $\mathcal{F}_{v}$ are the corresponding oriented foliations (whose leaves are the respective integral curves of $u$ and $v$ ), their geometric mean is the corresponding unoriented foliation $\mathcal{F}_{w}=\sqrt{\mathcal{F}_{u} \mathcal{F}_{v}}$ which bisects the angles formed by $\mathcal{F}_{u}$ and $\mathcal{F}_{v}$; a singularity of $\mathcal{F}_{w}$ is a singularity of $\mathcal{F}_{u}$ or $\mathcal{F}_{v}$.

By the rule of optics, "angle of incidence equals angle of reflection," the geometric mean is directly applicable to the definition of Euclidean conics by focal properties. The heuristics of this elementary application are considered in Sect. 2, where complex arithmetic and complex functions are seen to be very effective. In this context, the formula $w(z)=\sqrt{u(z) v(z)}$ for the geometric mean of vector fields may be taken literally; $u(z) v(z)$ is the product of complex functions, and the two-valued square root accounts for $w$ being unoriented. Likewise, the confocal family of conics $\mathcal{F}_{w}$ is unoriented (in fact, unorientable).

(c) The Author(s) 2018. This article is distributed under the terms of the Creative Commons Attribution 4.0 International License (http://creativecommons.org/licenses/by/4.0/), which permits unrestricted use, distribution, and reproduction in any medium, provided you give appropriate credit to the original author(s) and the source, provide a link to the Creative Commons license, and indicate if changes were made. 
For a fuller development of such ideas, the natural setting is provided by differentials on a Riemann surface $\mathcal{S}$. A meromorphic differential $\omega=f(z) \mathrm{d} z$ (dual to a meromorphic vector field $v=\frac{1}{f(z)} \frac{\partial}{\partial z}$ ) defines an oriented foliation $\mathcal{F}_{\omega}$ on $\mathcal{S}$, while a quadratic differential $Q=q(z) \mathrm{d} z^{2}$ defines an unoriented foliation $\mathcal{F}_{Q}$. If $\omega_{1}=f_{1}(z) \mathrm{d} z$ and $\omega_{2}=f_{2}(z) \mathrm{d} z$ define $\mathcal{F}_{\omega_{1}}$ and $\mathcal{F}_{\omega_{2}}$, then the product $Q=\omega_{1} \omega_{2}=f_{1}(z) f_{2}(z) \mathrm{d} z^{2}$ gives the geometric mean foliation $\mathcal{F}_{Q}=\sqrt{\mathcal{F}_{\omega_{1}} \mathcal{F}_{\omega_{2}}}$.

For our main application to conics, it suffices to consider the Riemann sphere $\mathcal{S} \simeq \hat{\mathbb{C}}=\mathbb{C} \cup\{\infty\}$, since the Euclidean plane $E^{2} \simeq \mathbb{C}$, the sphere $S^{2} \simeq \hat{\mathbb{C}}$ and the hyperbolic plane $H^{2} \simeq$ upper half plane\} may all be identified with subsets of $\hat{\mathbb{C}}$. In these conformal models, the isometries form subgroups of the Möbius group, and the lines (geodesics) are given by certain circle arcs in $\hat{\mathbb{C}}$.

Accordingly, oriented line pencils ("bundles of light rays") in $E^{2}, S^{2}$ and $H^{2}$ are represented by certain oriented circle pencils in $\hat{\mathbb{C}}$. In $S^{2}$, such a pencil is elliptic (e.g., outward rays from the origin); in $E^{2}$, it may be elliptic or parabolic (e.g., rightward horizontal lines); in $H^{2}$, it may be elliptic, parabolic or hyperbolic (e.g., counterclockwise circles centered at the origin). Note that the convenient term "oriented circle pencil" is slightly misleading in the elliptic case; each circle/line consists of two opposing arcs/rays (each of which is a leaf of the foliation).

Now differentials enter the picture as follows: an oriented circle pencil is the foliation $\mathcal{F}_{\omega}$ of some non-vanishing differential $\omega=f(z) \mathrm{d} z$ on $\hat{\mathbb{C}}$ (the residues of which determine the type of the pencil). Confocal families of conics are geometric means $\mathcal{F}_{Q}=\sqrt{\mathcal{F}_{\omega_{1}} \mathcal{F}_{\omega_{2}}}$ of certain pairs of such pencils. Because of the greater variety of pairs $\left\{\mathcal{F}_{\omega_{1}}, \mathcal{F}_{\omega_{2}}\right\}$ in $H^{2}$, the classification of conics in $H^{2}$ is considerably more complicated than in $E^{2}$ or $S^{2}$ (and thus is deferred to Part II).

Every confocal family of conics $\mathcal{F}_{Q}$ may be represented in closed form. In fact, such a non-vanishing quadratic differential $Q=\omega_{1} \omega_{2}$ has the form $Q=\frac{\mathrm{d} z^{2}}{p(z)}$, where $p(z)$ is a polynomial of degree $d \leq 4$, and $Q$ has four poles, counting multiplicities. Then, the socalled natural parameter $F(z)=\int_{a}^{z} \frac{\mathrm{d} w}{\sqrt{p(w)}}$ is generically an elliptic integral of the first kind; when $Q$ has a non-simple pole, $F(z)$ is elementary. Such an integral $F(z)$ may be inverted, giving parameterized trajectories of $\mathcal{F}_{Q}$ by elliptic (or elementary) functions.

Thus, the geometric mean provides not only a unified definition of conics, but also a computational method for their concrete representation. Further, the conformal setting in $\widehat{\mathbb{C}}$ and the grouping of conics into confocal families may be used to advantage in the discussion of equivalence and corresponding classification of conics.

Now we outline the contents of the paper. In view of our expository goals, familiarity with quadratic differentials on Riemann surfaces will not be assumed. For the case of $\mathcal{S}=\hat{\mathbb{C}}$ (where meromorphic differentials may be locally represented by rational functions), we provide a concrete and self-contained account of linear and quadratic meromorphic differentials and their foliations, in Sects. 3-5.

In Sect. 6, differentials are used to discuss the geometric mean foliation $\mathcal{F}_{Q}=\sqrt{\mathcal{F}_{1} \mathcal{F}_{2}}$ and its basic properties. As a first example, confocal families of Euclidean conics are revisited as meromorphic foliations. Pairs of elliptic pencils $\mathcal{F}_{1}, \mathcal{F}_{2}$ produce a confocal family of ellipses $\mathcal{F}_{Q}$ where $Q$ has simple poles at foci $f_{j}$ and a double pole at $\infty$. An elliptic-parabolic pencil pair produce a confocal family of parabolas $\mathcal{F}_{Q}$ where $Q$ has a simple pole at the focus and a triple pole at $\infty$. The parabolas are limits of ellipses; say, 
$f_{2} \rightarrow \infty$ (see Fig. 4). Confocal families of parabolas are parameterized via the squaring map $z^{2}$; ellipses/hyperbolas via $\sin z$.

As shown in Sect. 7, all spherical conics are ellipses belonging to confocal families $\mathcal{F}_{Q}=\sqrt{\mathcal{F}_{1} \mathcal{F}_{2}}$ defined by pairs of elliptical pencils. (The circles of such a pencil $\mathcal{F}_{j}$ pass through a focus and its antipode). Then, $Q$ has four simple poles and the spherical ellipses are parameterized by the Jacobi elliptic sine function $\operatorname{sn} z$. This provides a satisfying analogy to the parameterization of Euclidean ellipses by $\sin z$. In Part II, such ellipses are seen to satisfy quartic polynomial equations $p(x, y)=0$; as such, ellipses in elliptic geometry are in fact elliptic curves!

\section{Heuristics: conics via complex variables}

The primary task of the next several sections is to develop the tools to systematically handle orientable and non-orientable meromorphic foliations on the Riemann sphere. In this section, we present some simple heuristics which will help to motivate much of what follows.

Let $w=f(z)=u+i v$ be a meromorphic function on $\hat{\mathbb{C}}=\mathbb{C} \cup\{\infty\}$; that is, $f(z)=p(z) / q(z)$ is a rational function defined by polynomials $p, q$. We may also regard $f$ as a meromorphic vector field using the correspondence $u+i v \leftrightarrow u \hat{\mathbf{i}}+\nu \hat{\mathbf{j}}$. Naturally, the product of two given fields $f_{1}, f_{2}$ is another field $f_{1} f_{2}$-e.g., if $f$ is a field, so is its square $f^{2}$.

On the other hand, the square root of a field $\sqrt{f}$ is an object of a different type. In the polar form $f=r e^{i \theta}$, we have $\sqrt{f}=\sqrt{r} e^{i \theta / 2}$, where the value of the complex unit $\sigma=e^{i \theta / 2}$ at a (non-singular) point $z$ is defined only up to sign. But since both signs determine the same line through $z$, we may use $\sqrt{f}$ to define a line field $\mathcal{L}=\mathcal{L}(\sqrt{f})$. (Only $\sigma$ is directly recoverable from $\mathcal{L}$; but for suitable $\mathcal{L}$, the modulus $\sqrt{r}$ may also be recovered, up to a positive factor, by methods of complex analysis which we will not require).

We are now able to define the geometric mean of a pair of vector fields, $f_{j}=r_{j} e^{i \theta_{j}}, j=1,2$, to be the line field $\mathcal{L}=\mathcal{L}\left(\sqrt{f_{1} f_{2}}\right)$ defined as above from $\sqrt{f_{1} f_{2}}=\sqrt{r_{1} r_{2}} e^{i\left(\theta_{1}+\theta_{2}\right) / 2}$. Thus, The geometric mean $\mathcal{L}$ bisects the angle formed by $f_{1}, f_{2}$.

We now illustrate the above ideas using Euclidean conics. Consider the rule "angle of incidence equals angle of reflection" applied to a parabolic mirror-see Fig. 1 (left). The incident bundle of rays $\mathcal{I}$ departs from a light source at the parabola's focus. By a wellknown property of the parabola, the resulting reflected bundle $\mathcal{R}$ is a beam of horizontal rays directed to the right.

Extending the bundles $\mathcal{I}, \mathcal{R}$ to fill the whole plane, the above description applies simultaneously to all members of the family $\mathcal{F}$ of parabolas with the same focus and axis of symmetry-say, with focus $c=0$ and axis $y=0$-as shown in Fig. 1 (right). $\mathcal{R}$ may be said to solve the scattering problem for $\mathcal{F}$ with input $\mathcal{I}$.

But now we wish to regard both $\mathcal{I}$ and $\mathcal{R}$ as given, and to view $\mathcal{F}$ as the solution to the inverse-scattering problem: what are all the mirrors redirecting $\mathcal{I}$ to $\mathcal{R}$ (or $\mathcal{R}$ to $\mathcal{I}$ )? At each (regular) point $p, \mathcal{I}$ and $\mathcal{R}$ determine a unique line; namely, the line bisecting the angle they form at $p$. If $p$ is allowed to vary and $\mathcal{L}$ is the resulting line field, then $\mathcal{F}$ consists of integral curves of $\mathcal{L}$.

Note that in the above discussion there is no need for the speed of light to be constant! This fact will enable us, in the following examples, to use meromorphic vector fields $f_{1}, f_{2}$ to represent the bundles of rays $\mathcal{I}, \mathcal{R}$, and obtain $\mathcal{F}$ as the family of integral curves 


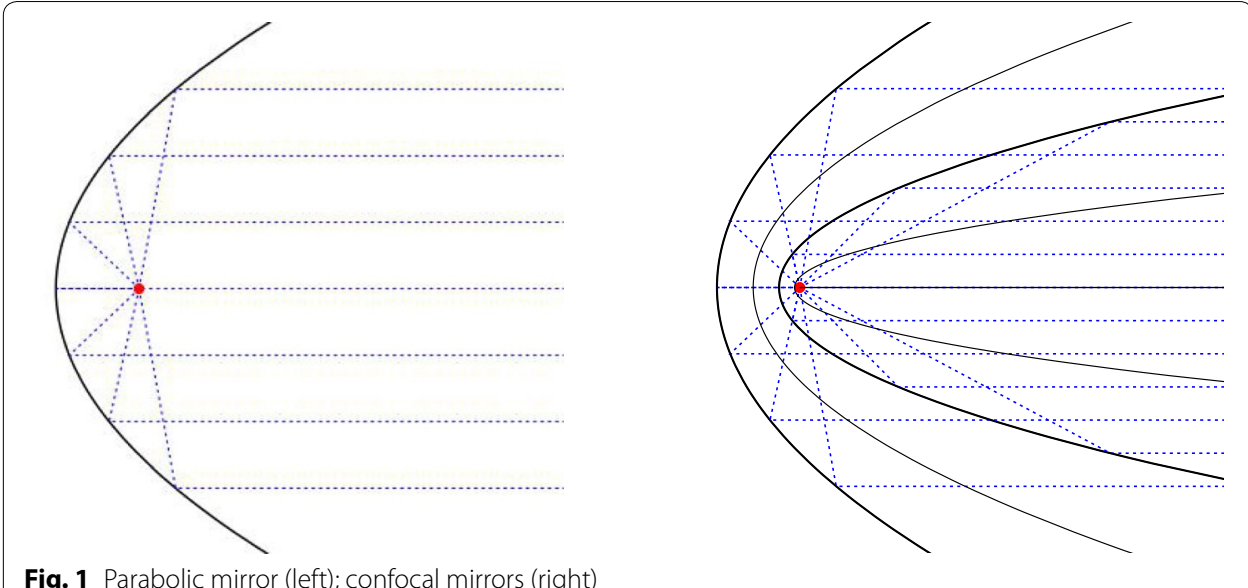

Fig. 1 Parabolic mirror (left); confocal mirrors (right)

of the geometric mean $\mathcal{L}\left(\sqrt{f_{1} f_{2}}\right)$. In practice, computing $\mathcal{F}$ amounts to formally solving the $\mathrm{ODE} \frac{\mathrm{d} z}{\mathrm{~d} t}=\sqrt{f_{1} f_{2}}$ by separation of variables, as we now illustrate.

Example 2.1 (Parabolas) In the above situation, the incident bundle of rays $\mathcal{I}$ may be represented by the outward radial vector field $f_{1}=z$. The reflected bundle $\mathcal{R}$ is represented by $f_{2}=1$. Thus, $\sqrt{f_{1} f_{2}}=\sqrt{z}$, and the confocal mirrors $\mathcal{F}$ solve the ODE: $\frac{\mathrm{d} z}{\mathrm{~d} t}=\sqrt{z}$.

By separation of variables, $\frac{\mathrm{d} z}{\sqrt{z}}=\mathrm{d} t$, we obtain $2 \sqrt{z}=t+i \nu_{0}$, hence, $z(t)=\left(t+i v_{0}\right)^{2} / 4$; here, we have chosen the convenient parameter $v_{0}$ giving the initial condition $z(0)=-v_{0}^{2} / 4$. These are confocal parabolas for $v_{0} \neq 0$; in particular, for $v_{0}>0$. Eliminating $t$ from $z=x+i y=\left(t^{2}-v_{0}^{2}\right) / 4+i v_{0} t / 2$ gives the quadric equations for $\mathcal{F}: v_{0}^{2} x=y^{2}-\frac{v_{0}^{4}}{4}$.

Changing signs of both $f_{1}, f_{2}$ leaves $\mathcal{F}$ unchanged, but reverses time in the optical interpretation: a leftwards horizontal beam reflects to rays converging at the focus (the focussing property of the parabola). Changing sign of exactly one of $f_{1}, f_{2}$ results in the orthogonal family of parabolas $z(t)=\left(\mu_{0}+i t\right)^{2} / 4$ - see Fig. 2 (left), in which the orthogonal foliations are superimposed.

Example 2.2 (Ellipses and hyperbolas): To parameterize confocal ellipses with foci $c_{1}=-1, \quad c_{2}=1$, recall the focussing property of ellipses: rays emitted by a light source at $c_{1}$ are reflected towards $c_{2}$.

The incident bundle $\mathcal{I}$ is represented by $f_{1}=z-c_{1}=z+1$ (outward radial vector field with center $c_{1}$ ). Similarly, the reflected bundle $\mathcal{R}$ is represented by $f_{2}=-\left(z-c_{2}\right)=1-z$ (inward radial vector field with center $c_{2}$ ). So ellipses satisfy the ODE: $\frac{\mathrm{d} z}{\mathrm{~d} t}=\sqrt{f_{1} f_{2}}=\sqrt{1-z^{2}}$.

Solution by separation of variables leads to ellipse parameterizations $z(t)=\sin \left(t+i \nu_{0}\right)=\cosh v_{0} \sin t+i \sinh v_{0} \cos t$. With $z=x+i y$, the confocal ellipses satisfy the quadric equations: $\frac{x^{2}}{\cosh ^{2} \nu_{0}}+\frac{y^{2}}{\sinh ^{2} \nu_{0}}=1$. Reversing sign of $f_{1}$ (or $f_{2}$ ) results in the confocal family of hyperbolas $z(t):=\sin \left(\mu_{0}+i t\right)=\sin \mu_{0} \cosh t+i \cos \mu_{0} \sinh t$ -Fig. 2 (right).

The above examples guide our treatment of non-Euclidean conics. In particular, we will address the following two questions. First, how should conics be defined, in a unified 


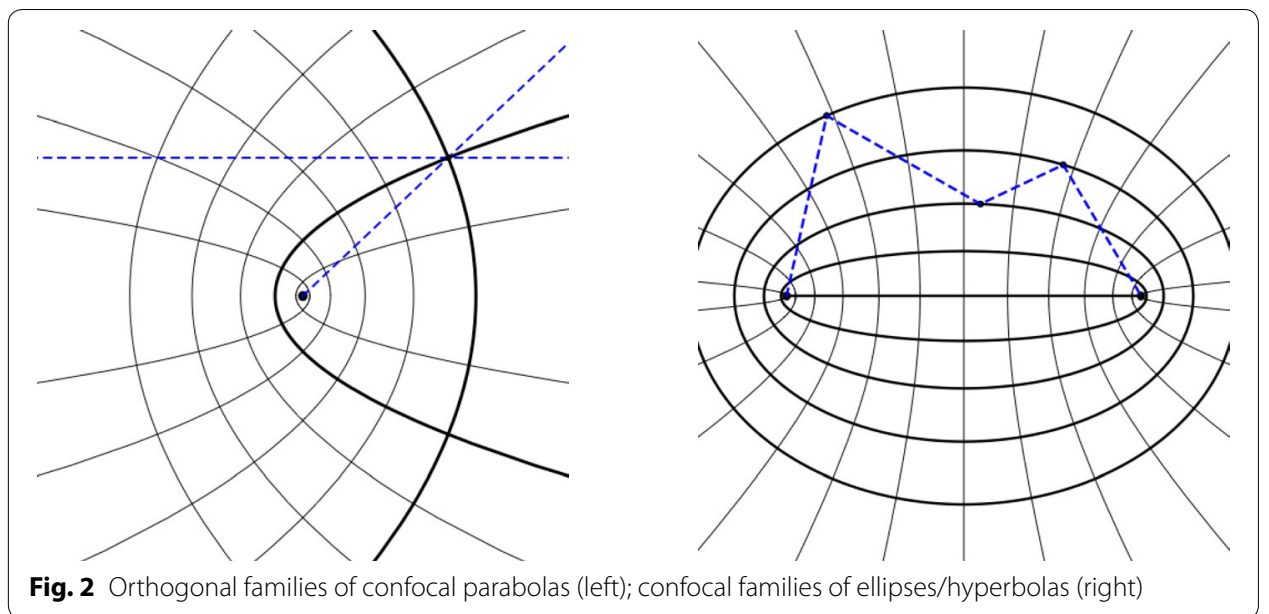

way which includes all possible types? Second, how are parameterizations for such conics computed?

So far we have appealed to familiar geometrical and dynamical ideas associated with vector fields, which will continue to serve heuristic purposes. But quadratic differentials turn out to be much better suited to the main task at hand. Thus, we proceed now to develop the basics of (linear and quadratic) differentials on the Riemann sphere. The Riemann sphere is the relevant domain, and limiting the discussion to this case has a considerable advantage of concreteness, which we will systematically exploit.

\section{Meromorphic differentials on the Riemann sphere}

The extended complex plane $\hat{\mathbb{C}}=\mathbb{C} \cup\{\infty\}$ is given the structure of a Riemann surface via local coordinates $z=x+i y$ on $\mathbb{C}$ and $\zeta=\varphi(z)=\frac{1}{z}$ on $\hat{\mathbb{C}} \backslash\{0\}$; at the point $z=\infty$, the limiting value $\zeta=\lim _{z \rightarrow \infty} \varphi(z)=0$ is assigned. On the overlapping domain $\mathbb{C}^{\times}=\mathbb{C} \backslash\{0\}$, the coordinate transition map $\varphi: \mathbb{C}^{\times} \rightarrow \mathbb{C}^{\times}$is conformal, as required. $\hat{\mathbb{C}}$ is identifiable with the unit sphere $S^{2} \subset \mathbb{R}^{3}$ via stereographic projection $\pi: S^{2} \rightarrow \hat{\mathbb{C}}$ from the north pole $n=\pi^{-1}(\infty)$. The Riemann sphere is the Riemann surface defined by the compatible coordinates $z$, $\zeta$ on $\hat{\mathbb{C}}$.

Remark 3.1 The full atlas for $\hat{\mathbb{C}}$ consists of $z_{1}=z, z_{2}=\zeta$, together with all compatible local coordinates. Any two, $z_{j}, z_{k}$, are related by a conformal map $\varphi_{j k}=z_{j} \circ z_{k}^{-1}: U \rightarrow V$. We will introduce various meromorphic objects and data (differentials, poles, residues, etc.) via the standard coordinates $z$ and $\zeta$; it will be understood (and may be easily checked) that such notions are well defined, i.e., independent of local coordinate $z_{j}$. (For present purposes, invariant definitions are hardly justified).

A meromorphic function on $\hat{\mathbb{C}}$ is represented by a pair of rational functions $h(z), \tilde{h}(\zeta)$, either one of which determines the other:

$$
h(z)=\frac{\sum_{j=0}^{J} a_{j} z^{j}}{\sum_{k=0}^{K} b_{k} z^{k}} ; \quad \tilde{h}(\zeta)=h(1 / \zeta)=\zeta^{K-J} \frac{\sum_{j=0}^{J} a_{j} \zeta^{J-j}}{\sum_{k=0}^{K} b_{k} \zeta^{K-k}}
$$


$\left(a_{j}, b_{k} \in \mathbb{C}, b_{K} \neq 0, a_{J} \neq 0\right.$ unless $\left.h \equiv 0\right)$. Accordingly, meromorphic functions on $\hat{\mathbb{C}}$ may be denoted simply $h(z)$, with the understanding that the behavior of $h$ at the point $z=\infty$ is that of $\tilde{h}$ at $\zeta=0$.

For instance, the order of $h$ at $a \in \mathbb{C}$-denoted $\operatorname{ord}_{a} h=v_{a} h=m$-is the degree of the lowest order term in the Laurent series near $a, h(z)=\sum_{j=m}^{\infty} a_{j}(z-a)^{j}$. In the finite plane, $h$ has $J$ zeros and $K$ poles, counting multiplicities, so $\sum_{z \in \mathbb{C}} \operatorname{ord}_{z} h=J-K$. On the other hand, $\operatorname{ord}_{\infty} h:=\operatorname{ord}_{0} \tilde{h}=K-J$. Thus, on the Riemann sphere, a meromorphic function has as many zeros as poles: $Z_{h}-P_{h}=\sum_{z \in \hat{\mathbb{C}}} \operatorname{ord}_{z} h=0$. (The corresponding result holds on any compact Riemann surface).

Along much the same lines, a meromorphic differential on the Riemann sphere, $\omega=f(z) \mathrm{d} z=\tilde{f}(\zeta) \mathrm{d} \zeta$, is represented by a pair of rational functions $f(z), \tilde{f}(\zeta)$. Either coefficient function $f$ or $\tilde{f}$ determines the other, this time according to the rule familiar from change of variable for integrals: $\omega=f(z) \mathrm{d} z=f(\varphi(\zeta)) \varphi^{\prime}(\zeta) \mathrm{d} \zeta=f(1 / \zeta)\left(\frac{-\mathrm{d} \zeta}{\zeta^{2}}\right)$. In other words, $\tilde{f}(\zeta)=-\frac{f(1 / \zeta)}{\zeta^{2}}$. With this understanding, a single rational function suffices to denote a meromorphic differential: $\omega=f(z) \mathrm{d} z$.

The order of $\omega$ at a point is (well defined by) the order of the representing coefficient function at the point. Thus, for $a \in \mathbb{C}, \operatorname{ord}_{a} \omega=\operatorname{ord}_{a} f$ and $\operatorname{ord}_{\infty} \omega=\operatorname{ord}_{0} \tilde{f}=\operatorname{ord}_{\infty} f-2$. E.g., while $f(z)=\frac{1}{z}$ has a simple pole at $z=0$ and a simple zero at $z=\infty$, the differential $\omega=f(z) \mathrm{d} z=\frac{\mathrm{d} z}{z}=-\frac{\mathrm{d} \zeta}{\zeta}$ has two simple poles (and no zeros).

More generally, it follows that a meromorphic differential on $\hat{\mathbb{C}}$ has two more poles than zeros : $Z_{\omega}-P_{\omega}=\sum_{z \in \hat{\mathbb{C}}} \operatorname{ord}_{z} \omega=-2$. (The general formula $\sum_{z \in S} \operatorname{ord}_{z} \omega=2 g-2$ for a meromorphic differential $\omega$ on a compact surface $S$ of genus $g \geq 1$ is much less trivial to prove).

The residue of $\omega$ at $a \in \hat{\mathbb{C}}$ may be handled similarly, using coordinates $z$ and $\zeta$. Thus, e.g., $\omega=\frac{\mathrm{d} z}{z}=-\frac{\mathrm{d} \zeta}{\zeta}$ has residues $\operatorname{Res}(\omega ; 0)=1$ and $\operatorname{Res}(\omega ; \infty)=-1$. The fact that these sum to zero generalizes to any $\omega=f(z) \mathrm{d} z$. A large enough circle $C$ : $z=\operatorname{Re}^{i t}$ contains all finite poles of $f(z)$, and the Residue Theorem gives $\int_{C} f(z) \mathrm{d} z=2 \pi i \sum_{z \in \mathbb{C}} \operatorname{Res}(\omega ; z)$. Under the change of variable $z=1 / \zeta, C$ corresponds to the counterclockwise circle in the $\zeta$-plane, $\tilde{C}: \zeta=e^{-i t} / R$, so $\int_{C} f(z) \mathrm{d} z=\int_{\tilde{C}} \tilde{f}(\zeta) \mathrm{d} \zeta=-2 \pi i \operatorname{Res}(\omega ; \infty)$.

The neat conclusion $\sum_{z \in \hat{\mathbb{C}}} \operatorname{Res}(\omega ; z)=0$ (which generalizes to any compact Riemann surface) is hard to even discuss without recognizing that residues (like all contour integrals) truly belong to differentials, not functions. (The common notation $\operatorname{Res}(f ; z)$, which omits $\mathrm{d} z$, may account for some awkward definitions of "the residue of $f$ at $z=\infty$ " in some textbooks).

For our definition of conics, an essential role is played by the non-vanishing, meromorphic differentials on the Riemann sphere. To describe these, we take advantage of another exceptional feature of $\hat{\mathbb{C}}$, namely its large group of automorphisms. The three (complex) dimensional group of linear fractional transformations (Möbius group) $G=\left\{g(z)=\frac{a z+b}{c z+d}: a, b, c, d \in \mathbb{C}, a d-b c \neq 0\right\}$ consists of diffeomorphisms $g: \hat{\mathbb{C}} \rightarrow \hat{\mathbb{C}}$ which are in fact holomorphic (at every point, either $g$ or a composite of $g$ with $\varphi$ is analytic).

We can use symmetries $g \in G$ to make new differentials from a given one. Namely, the pull-back of $\omega=f(z) \mathrm{d} z$ by $z=g(\eta)$ is given by $g^{*} \omega=f(g(\eta)) g^{\prime}(\eta) \mathrm{d} \eta$. Naturally, pullback by $g \in G$ preserves orders and residues ( $g$ being a "global coordinate change"). 
We call two differentials equivalent, and write $\omega_{1} \simeq \omega_{2}$, if $\omega_{2}=g^{*} \omega_{1}$, for some $g \in G$. In the following result, a normal form $g^{*} \omega$ is expressed in terms of the standard coordinate: $g^{*} \omega=f(g(z)) g^{\prime}(z) \mathrm{d} z, z \in \mathbb{C}$.

Proposition 3.2 A non-vanishing meromorphic differential $\omega$ on the Riemann sphere satisfies exactly one of the following two descriptions:

1. $\omega$ has two simple poles, $c_{+} \in \mathbb{C}, c_{-} \in \hat{\mathbb{C}}$, and $\omega \simeq \lambda \frac{\mathrm{d} z}{z}$, where $\lambda=\operatorname{Res}\left(\omega, c_{+}\right)$. Depending on whether $c_{-}$is finite or infinite,

$$
\omega=\frac{\lambda\left(c_{+}-c_{-}\right) \mathrm{d} z}{\left(z-c_{+}\right)\left(z-c_{-}\right)}, \quad \text { or } \quad \omega=\frac{\lambda \mathrm{d} z}{\left(z-c_{+}\right)} .
$$

2. $\omega$ has a double pole at $c \in \hat{\mathbb{C}}$ and $\omega \simeq \frac{\mathrm{d} z}{z^{2}} \simeq \mathrm{d} z$. For some $A \in \mathbb{C}^{\times}$,

$$
\omega=\frac{A \mathrm{~d} z}{(z-c)^{2}}, \quad \text { or } \quad \omega=A \mathrm{~d} z
$$

depending on whether $c$ is finite or infinite.

Proof In each case, the form of $\omega$ itself follows from the given data.

In case (1), if $c_{-} \neq \infty$, one obtains $\omega=g^{*} \frac{\lambda \mathrm{d} z}{z}$ using $g(z)=\frac{z-c_{+}}{z-c_{-}}$. If $c_{-}=\infty$, use $g(z)=z-c_{+}$.

In case (2), if $c$ is finite, $g^{*} \omega=\frac{\mathrm{d} z}{z^{2}}$, where $g(z)=A z+c$. The equivalence $\mathrm{d} z \simeq \frac{\mathrm{d} z}{z^{2}}$ uses the map $g(z)=-\frac{1}{z}$. Finally, $\lambda \frac{\mathrm{d} z}{z}$ is not equivalent to $\mathrm{d} z$, since $g \in G$ must preserve orders.

Remark 3.3 For brevity, we forgo a detailed discussion of meromorphic vector fields $V=v(z) \frac{\partial}{\partial z}$ on $\hat{\mathbb{C}}$. But note that $\frac{\partial}{\partial z}$ in $V$ is the counterpart of $\mathrm{d} z$ in $\omega=f(z) \mathrm{d} z$. (The operator formalism was not required in Sect. 2 since we were able to work in a fixed coordinate; for more on the meaning and use of $\frac{\partial}{\partial z}$, see [6]). The 1-1 correspondence $\omega=f(z) \mathrm{d} z \leftrightarrow V=\frac{1}{f(z)} \frac{\partial}{\partial z}$, realizes duality: $\omega(V)=\mathrm{d} z\left(\frac{\partial}{\partial z}\right)=\frac{\partial z}{\partial z}=1$.

Duality swaps zeros of one object with poles of the other, and translates Proposition 3.2 into the following statement: An analytic vector field $V$ on $\widehat{\mathbb{C}}$ either has two zeros, and $V \simeq a z \frac{\partial}{\partial z}$, for some $a \in \mathbb{C}^{\times}$, or has one double zero, and $V \simeq \frac{\partial}{\partial z} \simeq z^{2} \frac{\partial}{\partial z}$. (Here, equivalence is defined by push-forward by $g \in G$ ).

The vector fields $\frac{\partial}{\partial z}, z \frac{\partial}{\partial z}, z^{2} \frac{\partial}{\partial z}$ form a basis for a Lie algebra with commutator bracket $[V, W]=\left[v \frac{\partial}{\partial z}, w \frac{\partial}{\partial z}\right]=\left(v w^{\prime}-w v^{\prime}\right) \frac{\partial}{\partial z}$, and this Lie algebra may be identified with $\mathfrak{g} \simeq \mathfrak{s l}(2, \mathbb{C})$ — the Lie algebra of $G$. In light of such remarks, it should not be surprising that non-vanishing meromorphic differentials on the Riemann sphere should play a distinguished role, below. 


\section{Oriented, meromorphic foliations on $\hat{\mathbb{C}}$}

An oriented, meromorphic foliation $\mathcal{F}$ may be defined, provisionally, as the phase portrait of a meromorphic vector field $V=v(z) \frac{\partial}{\partial z}$. The zeros and poles of $V$ account for the isolated singularities of $\mathcal{F}$. With respect to the coordinate $z$, the leaves of $\mathcal{F}$ are parameterized by solutions of the ODE: $\frac{\mathrm{d} z}{\mathrm{~d} t}=v(z)$, or $\frac{\mathrm{d} z}{v(z)}=\mathrm{d} t$.

In terms of the dual differential $\omega=f(z) \mathrm{d} z=\frac{\mathrm{d} z}{v(z)}$, the equation may be expressed: $\omega=f(z) \mathrm{d} z=\mathrm{d} t$. Thus, duality formalizes the separation of variables technique and explains the use of $\omega$, in place of $V$, for defining meromorphic foliations.

Given a meromorphic differential $\omega=f(z) \mathrm{d} z$, the corresponding oriented, meromorphic foliation will be denoted $\mathcal{F}=\mathcal{F}_{\omega}$. As the term suggests, the leaves of $\mathcal{F}$ are sometimes regarded as oriented (but not necessarily parameterized) curves. Then, $\mathcal{F}$ may be concisely defined by the condition of positivity $\omega>0$ : for any parameterization $z(t)$ of an oriented leaf, $f(z(t)) z^{\prime}(t)>0$ holds for all $t$ (likewise $\tilde{f}(\zeta(t)) \zeta^{\prime}(t)>0$ ).

Conversely, $\mathcal{F}$ determines $\omega=f(z) \mathrm{d} z$, up to scaling by a positive constant. For if $\omega_{1}=f_{1}(z) \mathrm{d} z$ gives the same oriented foliation, then $\omega_{1} / \omega=f_{1} / f=r$ is a well-defined, positive, meromorphic function $r(z)$. Such a function is locally constant.

The previous remarks notwithstanding, $\mathcal{F}_{\omega}$ will usually be understood here to consist of curves which are not merely oriented, but parameterized by $z(t)$ solving the ODE: $f(z(t)) z^{\prime}(t)=1$. Such distinguished parameterizations will be obtained in our explicit examples.

For this purpose, we fix some notation. Let $a$ be regular point of $\omega=f(z) \mathrm{d} z$. Then, $f$ has a local, analytic antiderivative $F(z):=\int_{a}^{z} f(w) \mathrm{d} w$; the integral may be taken along straight line paths from $a$ to $z$, a nearby point. (In other words, a meromorphic differential $\omega$ is closed, i.e., locally exact: near $a$, we can always write $f \mathrm{~d} z=\mathrm{d} F$ ). Further, since $a$ is a regular point, we can also define the local inverse $\phi=F^{-1}$. For $z_{0}=\phi\left(t_{0}\right)$ in the image of $\phi$, we may define $z(t):=\phi\left(t+t_{0}\right)$ for small $t$. Then, $z(t)$ solves the initial value problem: $\frac{\mathrm{d} z}{\mathrm{~d} t}=\frac{1}{f(z)}, z(0)=z_{0}$.

Example 4.1 We describe $\mathcal{F}_{\omega}$ for $\omega=\lambda \frac{\mathrm{d} z}{z}, \frac{\mathrm{d} z}{z^{2}}, \mathrm{~d} z$ (using $t_{0}=0$ ):

1. $\mathcal{F}_{\lambda \mathrm{d} z / z}: z(t)=z_{0} e^{t / \lambda}, z_{0} \neq 0$, are rays, circles or logarithmic spirals, depending on whether $\lambda$ is real, imaginary, or neither. Transferred to $S^{2} \simeq \hat{\mathbb{C}}, \mathcal{F}$ consists of meridians, parallels of latitude, or $\theta$-loxodromes, with singularities at the poles $n$, $s$.

2. $\mathcal{F}_{\mathrm{d} z / z^{2}}: z(t)=\frac{z_{0}}{1-t z_{0}}, z_{0} \neq 0$, are circles tangent to the real axis at $z=0$, where they form a dipole singularity.

$3 \mathcal{F}_{\mathrm{d} z}: z(t)=z_{0}+t$ are horizontal lines-the stereographic images of circles on $S^{2}$ through $n$, tangent to the circle $(\cos t, 0, \sin t)$.

Any foliation of a non-vanishing $\omega$ looks like one in the example, since equivalence of differentials $\omega_{1} \simeq \omega_{2}$ implies equivalence of foliations $\mathcal{F}_{\omega_{1}} \simeq \mathcal{F}_{\omega_{2}}$; that is, the leaves of $\mathcal{F}_{\omega_{1}}$ are carried to those of $\mathcal{F}_{\omega_{2}}$ by some $g \in G$. (E.g., the dipole singularity at $z=0$ in (2) is related by $z \mapsto-1 / z$ to the dipole at $z=\infty$ in (3)). Conversely: $\mathcal{F}_{\omega_{1}} \simeq \mathcal{F}_{\omega_{2}} \Rightarrow \omega_{1} \simeq r \omega_{2}$ for some $r>0$.

In order to describe such special foliations in more geometric terms, we recall some terminology. An elliptic pencil consists of all the circle arcs/lines terminating at a given 
pair of singular points $k_{ \pm} \in \hat{\mathbb{C}} ; k_{ \pm}$are also the limit points of the hyperbolic pencil of circles orthogonal to any given circle in the elliptic pencil. A parabolic pencil contains all the circles tangent to a given line $L_{0}$ at the dipole $p_{0}$; or the pencil consists of parallel lines (with dipole at $p_{0}=\infty$ ). A $\theta$-loxodromic foliation with singularities $k_{ \pm}$consists of curves making a constant angle $\theta$ with the circles through $k_{ \pm}$.

Since any $g \in G$ takes a pencil or loxodromic foliation to another of the same type (and preserves $\theta$ for a loxodromic foliation), the following proposition is mostly a translation of Proposition 3.2.

Proposition 4.2 The foliation $\mathcal{F}=\mathcal{F}_{\omega}$ of a non-vanishing meromorphic differential $\omega$ on $\hat{\mathbb{C}}$ has either one or two singularities. In the former case, $\mathcal{F}$ is a parabolic pencil. Otherwise, $\mathcal{F}$ is an elliptic or hyperbolic pencil, or a loxodromic foliation. All pencils of a given type are equivalent, as are all $\theta$-loxodromic foliations for a given $\theta$. Any two circles determine a unique pencil; any loxodrome determines a unique loxodromic foliation.

Proof For $\omega=\frac{A \mathrm{~d} z}{(z-c)^{2}} \simeq \frac{\mathrm{d} z}{z^{2}}, \mathcal{F}_{\omega}$ is the parabolic pencil of circles tangent to the line $\gamma(t)=A t+c$ at $c$. If $\omega=A \mathrm{~d} z, \mathcal{F}_{\omega}$ is the pencil of lines parallel to $\gamma(t)=\frac{t}{A}$ and the dipole singularity lies at $z=\infty$.

If $\omega$ has poles $c_{ \pm} \in \hat{\mathbb{C}}$. then either:

a) $\lambda \in \mathbb{R}$ and $\mathcal{F}_{\omega}$ is the elliptic pencil of circles/lines through $c_{ \pm}$,

b) $\lambda \in i \mathbb{R}$ and $\mathcal{F}_{\omega}$ is the hyperbolic pencil with limit points $c_{ \pm}$, or

c) $\lambda=r e^{i \theta}$ and $\mathcal{F}_{\omega}$ is $\theta$-loxodromic with singularities $c_{ \pm}$.

Let $\mathcal{C}$ be the family of all circles and lines in $\hat{\mathbb{C}}$. The pencil $\overline{C_{1} C_{2}}$ is elliptic, parabolic, or hyperbolic, respectively, depending on whether $\left\{C_{1}, C_{2}\right\}$ have two, one, or zero points of intersection in $\hat{\mathbb{C}}$. In the first two cases, $\omega$ is easily constructed from Proposition 3.2. In the last case, the required differential is $i \omega$, where $\omega$ defines the elliptic pencil $\overline{O_{1} O_{2}}$ of orthogonal circles to $\left\{C_{1}, C_{2}\right\}$; in fact, one can take $O_{1}, O_{2}$ to be the circles whose diameters are tangent to $\left\{C_{1}, C_{2}\right\}$ at endpoints and, by geometry, $O_{1}, O_{2}$ intersect in two distinct points.

We conclude this section with some additional terminology which places the proposition in a somewhat broader context.

Given a meromorphic differential $\omega=f(z) \mathrm{d} z$, the equation $\frac{\mathrm{d} z}{\mathrm{~d} t}=\frac{1}{f(z)}$ may be regarded as an ODE in the complex domain. Formally, we simply replace $t$ by "complex time" $\tau=\mu+i v$ and let $\frac{\mathrm{d}}{\mathrm{d} \tau}$ be the complex derivative. But now solutions $z(\tau)=\phi\left(\tau+\tau_{0}\right)$ are not parameterized curves but local conformal maps. The curves can be recovered as restrictions of $\phi$ to horizontal lines, $z(t):=\phi\left(t+\tau_{0}\right), \tau_{0} \in \mathbb{C},-\epsilon<t<\epsilon$. For this reason, such trajectories may also be called rightward trajectories of $\omega$.

Likewise, the curves $z(t):=\phi\left(i t+\tau_{0}\right)$, which make up the orthogonal foliation to $\mathcal{F}_{\omega}$, may be called upward trajectories of $\omega$. More generally, for a given angle $\theta \in \mathbb{R}$, a curve $z(t):=\phi\left(t e^{i \theta}+\tau_{0}\right)$ is a $\theta$-trajectory of $\omega$. By the chain rule, such a curve satisfies $\frac{\mathrm{d} z}{\mathrm{~d} t}=e^{i \theta} / f(z)$, namely the equation satisfied by the (rightward) trajectories of the differential $\omega_{\theta}=e^{-i \theta} \omega$. Thus, $\theta$-trajectories of $\omega$ define the foliation $\mathcal{F}_{\omega_{\theta}}$. 
For a given foliation $\mathcal{F}_{\omega}$, we refer to $\left\{\mathcal{F}_{\omega_{\theta}}\right\}_{\theta \in \mathbb{R}}$ as the associated family of foliations. Note that all foliations in Proposition 4.2 with the same singular set belong to the same associated family. Also, up to conformal equivalence, there are only two such associated families, $\left\{\mathcal{F}_{e^{-i \theta} \mathrm{d} z}\right\}_{\theta \in \mathbb{R}}$ and $\left\{\mathcal{F}_{e^{-i \theta} \mathrm{d} z / z}\right\}_{\theta \in \mathbb{R}}$-the parabolic one and the other one!

\section{Quadratic differentials and non-orientable foliations}

A meromorphic quadratic differential $Q=q(z) \mathrm{d} z^{2}=\tilde{q}(\zeta) \mathrm{d} \zeta^{2}$ on $\widehat{\mathbb{C}}$ is given by a pair of rational functions, $q(z), \tilde{q}(\zeta)$, with $z=\varphi(\zeta)=\frac{1}{\zeta}$; the rule relating $q$, $\tilde{q}$ makes $Q$ transform like the square of a linear differential: $\tilde{q}(\zeta)=q(\varphi(\zeta))\left(\varphi^{\prime}(\zeta)\right)^{2}=q\left(\frac{1}{\zeta}\right) \frac{\mathrm{d} \zeta^{2}}{\zeta^{4}}$.

In case $Q=q(z) \mathrm{d} z^{2}$ actually is the square of a meromorphic differential, it is called orientable. Thus, e.g., $Q_{1}=\mathrm{d} z^{2}=\left(\frac{-\mathrm{d} \zeta}{\zeta^{2}}\right)^{2}$ is orientable, while $Q_{2}=z \mathrm{~d} z^{2}=\frac{\mathrm{d} \zeta^{2}}{\zeta^{5}}$ is non-orientable, since $\sqrt{q(z)}=\sqrt{z}$ is not meromorphic near odd order points. In fact, $Q=\frac{a(z)}{b(z)} \mathrm{d} z^{2}$ is orientable precisely when it has even order at all points; this follows from factorization of polynomials $a(z), b(z)$.

Examples will shortly explain the reason for the above nomenclature. In the meantime, we briefly review elements of the general theory, heuristically treating any $Q=q(z) \mathrm{d} z^{2}$ as if it were orientable. In fact, we may write $Q=\omega^{2}=(\sqrt{z} \mathrm{~d} z)^{2}$ even when $\omega=\sqrt{z} \mathrm{~d} z$ is only locally defined, up to sign, and in any case fails to be meromorphic at points of odd order.

First, a quadratic differential on $\widehat{\mathbb{C}}$ satisfies $\sum_{z \in \hat{\mathbb{C}}}$ ord $_{z} Q=-4$; for instance, $Q_{1}$ (above) has no zeros and one fourth order pole at $\zeta=0$, while $Q_{2}$ has a simple zero at $z=0$ and a fifth order pole at $\zeta=0$. In fact, the proof is the same as the one given earlier for differentials. (For genus $g>0$, the expected formula holds: $\sum_{z \in S} \operatorname{ord}_{z} Q=4 g-4$ ).

Next, a meromorphic quadratic differential $Q=q(z) \mathrm{d} z^{2}$ determines a meromorphic foliation $\mathcal{F}_{Q}$ by the positivity condition $Q>0$; that is, $q(z(t))\left(z^{\prime}(t)\right)^{2}>0$ is required to hold along trajectories (likewise $\tilde{q}(\zeta(t))\left(\zeta^{\prime}(t)\right)^{2}>0$ ). However, the foliation $\mathcal{F}_{Q}$ is unoriented, the condition being unaffected by reversal of orientation of $z(t)$. In the case of an orientable quadratic differential $Q=\omega^{2}$, we may also abuse notation and write $\mathcal{F}_{Q}=\mathcal{F}_{\omega}$ - simply neglecting orientation of the latter, since $\omega^{2}=(-\omega)^{2}$.

Not only does $Q$ determine $\mathcal{F}_{Q}$, we also know that $\mathcal{F}_{Q_{1}}=\mathcal{F}_{Q_{2}} \Rightarrow Q_{1}=r Q_{2}$, for some positive constant $r$, by the same argument as before. To summarize: quadratic differentials are to unoriented foliations as (linear) differentials are to oriented foliations.

Next, we define distinguished parameterizations $z(t)$ in the non-orientable case. Let $a$ be a regular point of a quadratic differential $Q=q(z) \mathrm{d} z^{2}$. The so-called natural parameter (or distinguished parameter) for $Q$ is locally defined, up to sign, by the integral

$$
\tau=F(z)=\int_{a}^{z} \sqrt{Q}=\int_{a}^{z} \sqrt{q(w)} \mathrm{d} w
$$

where $\sqrt{q(z)}$ is an analytic branch of the square root of $q(z)$ near $a$.

Then, $\tau=F(z)$ is a local conformal map, with local inverse $\phi(\tau)$ satisfying the ODE: $q(\phi(\tau))\left(\phi^{\prime}(\tau)\right)^{2}=1$. Further, for $z_{0}=\phi\left(\tau_{0}\right)$ in the image of $\phi$. we may define $z(t):=\phi\left(t+\tau_{0}\right)$ for $-\epsilon<t<\epsilon$. Then, $z(t)$ solves the initial value problem:

$$
\frac{\mathrm{d} z}{\mathrm{~d} t}=\frac{1}{\sqrt{q(z)}}, z(0)=z_{0}=\phi\left(\tau_{0}\right) .
$$


The reverse orientation $z(-t)$ solves the ODE for the opposite sign of $\sqrt{q(z)}$, and again we see that both orientations $z( \pm t)$ satisfy the condition $q(z)\left(z^{\prime}\right)^{2}=1>0$ for $Q$ trajectories.

The term horizontal trajectory is also used to refer to $z(t)$ (or the corresponding unparameterized, unoriented curve), since it is mapped by $F$ to a horizontal line in the $\tau$ plane. Likewise, $z(t):=\phi\left(i t+\tau_{0}\right)$ satisfies $q(z)\left(z^{\prime}\right)^{2}=-1<0$, and is called a vertical trajectory of Q. More generally, $z(t):=\phi\left(t e^{i \theta}+\tau_{0}\right)$ satisfies $q(z)\left(z^{\prime}\right)^{2}=e^{2 i \theta}$ and is a called a $\theta$-trajectory of $Q$. The same equation describes the (horizontal) trajectories of $Q_{2 \theta}=e^{-2 i \theta} Q$, thus, $\theta$-trajectories of $Q$ give the foliation $\mathcal{F}_{Q_{2 \theta}}$. For a given foliation $\mathcal{F}_{Q}$ we refer to $\left\{\mathcal{F}_{Q_{2 \theta}}\right\}_{0 \leq \theta<\pi}$ as the associated family of foliations.

Now we come to difference between the orientable and non-orientable cases, which is illustrated in Fig. 3. Unlike the hyperbolic pencil of circles (left) for $Q=-\frac{\mathrm{d} z^{2}}{z^{2}}=\left(\frac{i \mathrm{~d} z}{z}\right)^{2}$, the foliation of $Q=\frac{\mathrm{d} z^{2}}{z}$ (middle) allows no consistent orientation for its trajectories (in any neighborhood of the simple pole $z=0$ ). We will soon return to this non-orientable foliation, which may be recognized as the family of confocal parabolas of Example 8.

Likewise, the foliation of $Q=z \mathrm{~d} z^{2}$ (right) allows no consistent orientation (in any neighborhood of the simple zero $z=0$ ). We may nevertheless write $F(z)=\int \sqrt{z} \mathrm{~d} z=\frac{2}{3} z^{3 / 2}$ and parameterize trajectories by $z(t)=\phi\left(t+\tau_{0}\right)=\left(\frac{3}{2}\right)^{2 / 3}\left(t+\tau_{0}\right)^{2 / 3}$. In particular, for real values of $\tau_{0}$, the multi-valued function $z(t)$ parameterizes the three critical rays: $\arg z=0, \frac{2 \pi}{3}, \frac{4 \pi}{3}$. Similarly, $(n+2)$ critical trajectories meet at a zero of order $n$, so a zero of odd order results in a non-orientable foliation.

To summarize, odd order zeros and poles result in line field singularities (as distinguished from vector field singularities), and their presence accounts for the greater variety of foliations of quadratic differentials as compared with linear differentials (or vector fields). For more background on quadratic differentials and their foliations, see [6] and [11].

\section{Geometric means of oriented, meromorphic foliations}

The product of two linear differentials $\omega_{1}=f_{1}(z) \mathrm{d} z$ and $\omega_{2}=f_{2}(z) \mathrm{d} z$ is the well-defined quadratic differential $Q=\omega_{1} \omega_{2}=f_{1}(z) f_{2}(z) \mathrm{d} z^{2}$. This makes possible the following definition.

Definition 6.1 Let $\omega_{1}, \omega_{2}$ be meromorphic differentials and let $Q=\omega_{1} \omega_{2}$ be their product. Then, the geometric mean of oriented foliations $\mathcal{F}_{1}=\mathcal{F}_{\omega_{1}}$ and $\mathcal{F}_{2}=\mathcal{F}_{\omega_{2}}$ is the unoriented foliation $\mathcal{F}=\mathcal{F}_{Q}$. The notation $\mathcal{F}=\sqrt{\mathcal{F}_{1} \mathcal{F}_{2}}$ will also be used.

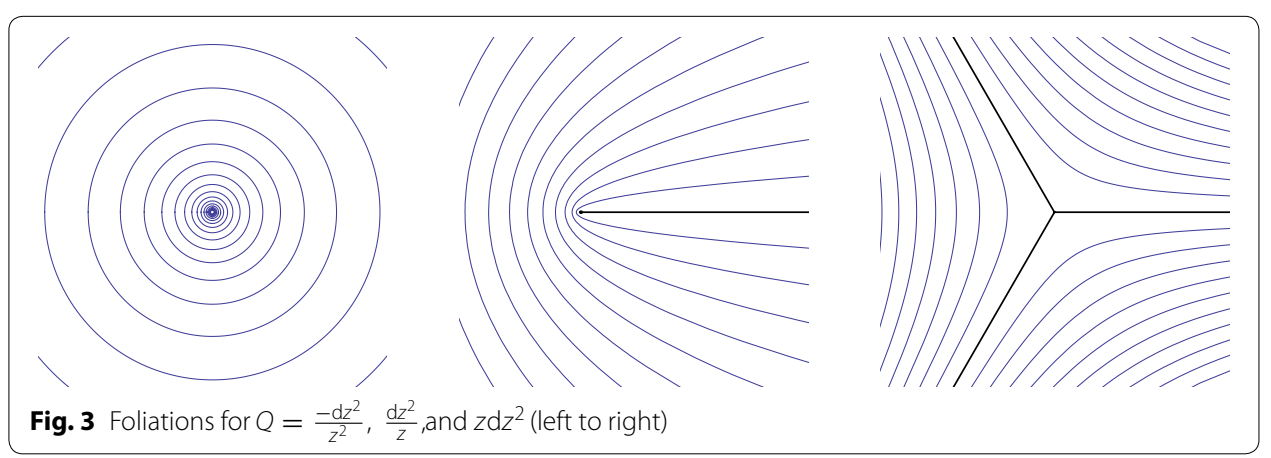


Let the following two remarks suffice to heuristically justify the above terminology and notation. First, let $Q_{j}=\omega_{j}^{2}$ (so $\mathcal{F}_{j}=\mathcal{F}_{Q_{j}}$, as unoriented foliations). Then, $Q^{2}=Q_{1} Q_{2}$ (as quartic differentials), suggesting the appealing (if slightly misleading) notation $\sqrt{Q_{1} Q_{2}}:=Q=\omega_{1} \omega_{2}$, hence, $\sqrt{\mathcal{F}_{1} \mathcal{F}_{2}}=\mathcal{F}_{\sqrt{Q_{1} Q_{2}}}$.

Second, we relate the above notion to the geometric mean of vectors, as defined in Sect. 2. For $j=1,2$, let $z_{j}(t)$ be the trajectory of $\mathcal{F}_{j}$ through $z_{0}$; that is, $z_{j}$ satisfies $\frac{\mathrm{d} z}{\mathrm{~d} t}=\frac{1}{f_{j}(z)}, z(0)=z_{0}$. Likewise, let $z(t)$ be a trajectory of $\mathcal{F}$, satisfying Eq. 2 for $q=f_{1} f_{2}$. Further, let $v_{j}=z_{j}^{\prime}(0)$ and $v=z^{\prime}(0)$. Then, the equations $1=f_{1} v_{1}=f_{2} v_{2}=f_{1} f_{2} v^{2}$ imply $v^{2}=v_{1} v_{2}$, so the line $\mathcal{L}(v)=\mathcal{L}\left(\sqrt{v_{1} v_{2}}\right)$ is the geometric mean of vectors $v_{1}, v_{2}$. To summarize:

Proposition 6.2 Let $\mathcal{F}=\sqrt{\mathcal{F}_{1} \mathcal{F}_{2}}$ be the geometric mean of oriented foliations $\mathcal{F}_{1}$ and $\mathcal{F}_{2}$. Then, the (unoriented) trajectories of $\mathcal{F}$ bisect the angles formed by the trajectories of $F_{1}$ and $F_{2}$.

Remark 6.3 Reversal of orientation of exactly one of $\mathcal{F}_{1}$ and $\mathcal{F}_{2}$ changes $\mathcal{F}_{Q}=\sqrt{\mathcal{F}_{1} \mathcal{F}_{2}}$ to the orthogonal foliation $\mathcal{F}_{-Q}$ (the vertical foliation of $Q$ ); orientation-reversal of both $\mathcal{F}_{1}, \mathcal{F}_{2}$ leaves $\mathcal{F}_{Q}$ unchanged. One could define the geometric mean of two unoriented (but orientable) foliations $\mathcal{F}_{\omega_{1}^{2}}, \mathcal{F}_{\omega_{2}^{2}}$ as the orthogonal pair of foliations $\mathcal{F}_{ \pm \omega_{1} \omega_{2}}$. But since many of our applications to conics require us to distinguish $\mathcal{F}_{Q}$ from $\mathcal{F}_{-Q}$, we will not make use of such a definition here.

Proposition 6.2 puts us in position to apply the geometric mean to confocal families of conics in the Euclidean plane $E^{2} \simeq \mathbb{C}$. However, we sidestep the loaded terms confocal and conic, for the moment, in favor of mirror foliation. Although we have yet to discuss lines (geodesics) in the conformal models of the sphere $S^{2}$ or hyperbolic plane $H^{2}$, we now make a definition which will apply to all models $M^{2}=E^{2}, S^{2}, H^{2}$ :

Definition 6.4 An oriented line pencil in $M^{2}$ is an oriented circle pencil in $\hat{\mathbb{C}}$ whose leaves represent geodesics in $M^{2}$. A mirror foliation in $M^{2}$ is the geometric mean foliation $\mathcal{F}=\sqrt{\mathcal{F}_{1} \mathcal{F}_{2}}$ of a pair of oriented line pencils $\mathcal{F}_{1}, \mathcal{F}_{2}$ in $M^{2}$.

Example 6.5 (Linear/circular mirrors in $E^{2}$ ) Leftward rays are reflected to rightward rays by vertical linear mirrors: $\sqrt{\mathcal{F}_{-\mathrm{d} z} \mathcal{F}_{\mathrm{d} z}}=\mathcal{F}_{-\mathrm{d} z^{2}}$. Rightward rays continue uninterrupted by horizontal linear mirrors: $\sqrt{\mathcal{F}_{\mathrm{d} z} \mathcal{F}_{\mathrm{d} z}}=\mathcal{F}_{\mathrm{d} z^{2}}$. More generally, two families of parallel rays are related by bisecting linear mirrors: $Q=e^{-i \theta_{1}} \mathrm{~d} z e^{-i \theta_{2}} \mathrm{~d} z=e^{-i\left(\theta_{1}+\theta_{2}\right)} \mathrm{d} z^{2}$.

Rays from $z_{0} \in \mathbb{C}$ are reflected back to $z_{0}$ by the concentric circular mirrors $\left|z-z_{0}\right|=R: Q=\frac{\mathrm{d} z}{z-z_{0}} \frac{-\mathrm{d} z}{z-z_{0}}=\frac{-\mathrm{d} z^{2}}{\left(z-z_{0}\right)^{2}}$. Note these linear and circular mirror foliations are orientable (but unoriented, by definition).

Example 6.6 (Confocal parabolas revisited) The incident bundle of rays from the origin make up the elliptic pencil $\mathcal{I}=\mathcal{F}_{\mathrm{d} z / z}$; the reflected rays give the parabolic pencil $\mathcal{R}=\mathcal{F}_{\mathrm{d} z}$. The geometric mean $\mathcal{F}_{Q}=\sqrt{\mathcal{I} \mathcal{R}}$ is defined by $Q=\frac{\mathrm{d} z^{2}}{z}=\frac{\mathrm{d} \zeta^{2}}{\zeta^{3}}$; this is the foliation by confocal parabolas, $z(t)=\left(t+i \nu_{0}\right)^{2} / 4$ (solving Eq. 2, that is, $z^{\prime 2}=z$ with $\tau_{0}=i \nu_{0}$ ). Since $Q$ has a simple pole at $z=0$ and a triple pole at $z=\infty, \mathcal{F}_{Q}$ is non-orientable. 


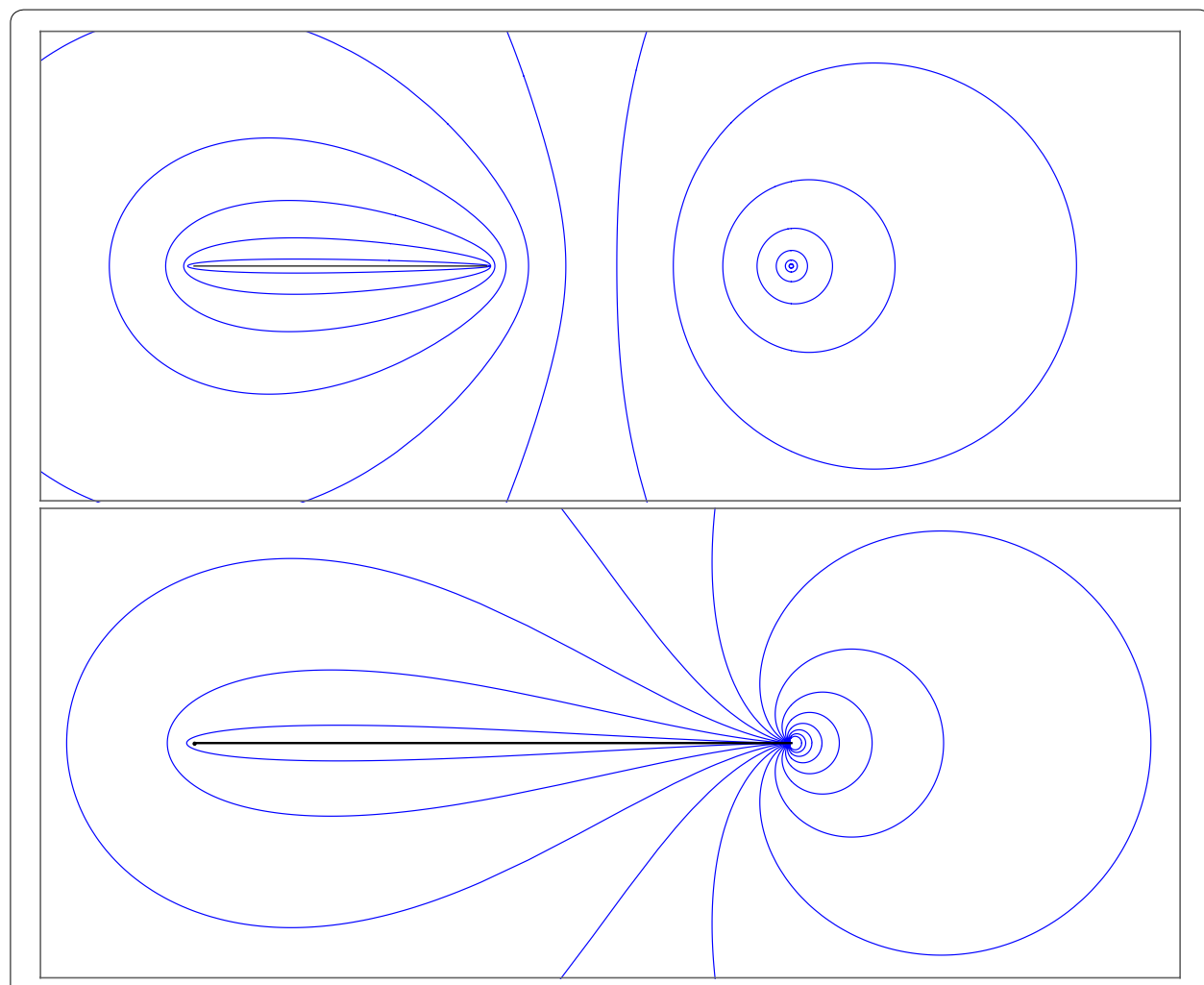

Fig. 4 Conformal images of confocal ellipses (top) and confocal parabolas (bottom), with $\infty \mapsto 1$

But now we wish to get a more global view of $\mathcal{F}_{Q}$, showing also the triple pole at $z=0$. Namely, we map by $g(z)=\frac{z-1}{z+1}$, which satisfies $g(0)=-1, g(\infty)=1$. The resulting family of curves $\tilde{z}(t)=g(z(t))=\frac{\left(t+i \nu_{0}\right)^{2}-4}{\left(t+i v_{0}\right)^{2}+4}$ shows singularities at $z= \pm 1$-see Fig. 4, bottom (and compare Fig. 3, middle).

Example 6.7 (Confocal ellipses revisited) The confocal ellipses reflect rays $\mathcal{I}$ from focus $c_{1}=-1$ to rays $\mathcal{R}$ which converge at the other focus $c_{2}=1$. These elliptic pencils are defined by $\omega_{1}=\frac{\mathrm{d} z}{1+z}$ and $\omega_{2}=\frac{\mathrm{d} z}{1-z}$. The geometric mean foliation is again non-orientable, since it is defined by $Q=\frac{\mathrm{d} z^{2}}{1-z^{2}}=\frac{\mathrm{d} \zeta^{2}}{\zeta^{2}\left(1-\zeta^{2}\right)}$, which has simple poles at $z= \pm 1$ and a double pole at $z=\infty$.

$\mathcal{F}_{Q}$ was already shown in Example 2.2, where we obtained the confocal ellipse parameterizations: $z(t)=\sin \left(t+i v_{0}\right)^{2}$. For a view of $\mathcal{F}_{Q}$ showing also the double pole at $z=\infty$, we map by $g(z)=\frac{z-1}{z+3}$, which satisfies $g(-1)=-1, g(1)=0, g(\infty)=1$. The resulting family of curves $\tilde{z}(t)=g(z(t))=\frac{\sin \left(t+i \nu_{0}\right)-1}{\sin \left(t+i \nu_{0}\right)+3}$ shows singularities at $z=0, \pm 1-$ see Fig. 4 (top).

By comparing the top and bottom of the figure, one sees the parabola family as a limit of the ellipse family, where the simple pole of the latter at $z=0$ slides to the right until it collides with the double pole, resulting in a triple pole at $z=1$. This correspond to the limit $\lim _{c \rightarrow 1} \frac{-2 \mathrm{~d} z^{2}}{(z-c)(z+1)(z-1)^{2}}=\frac{2 \mathrm{~d} z^{2}}{(z+1)(1-z)^{3}}$, which coincides with the $\tilde{Q}$ for parabolas (up to a factor of 2). So parabolas are limits of ellipses! 

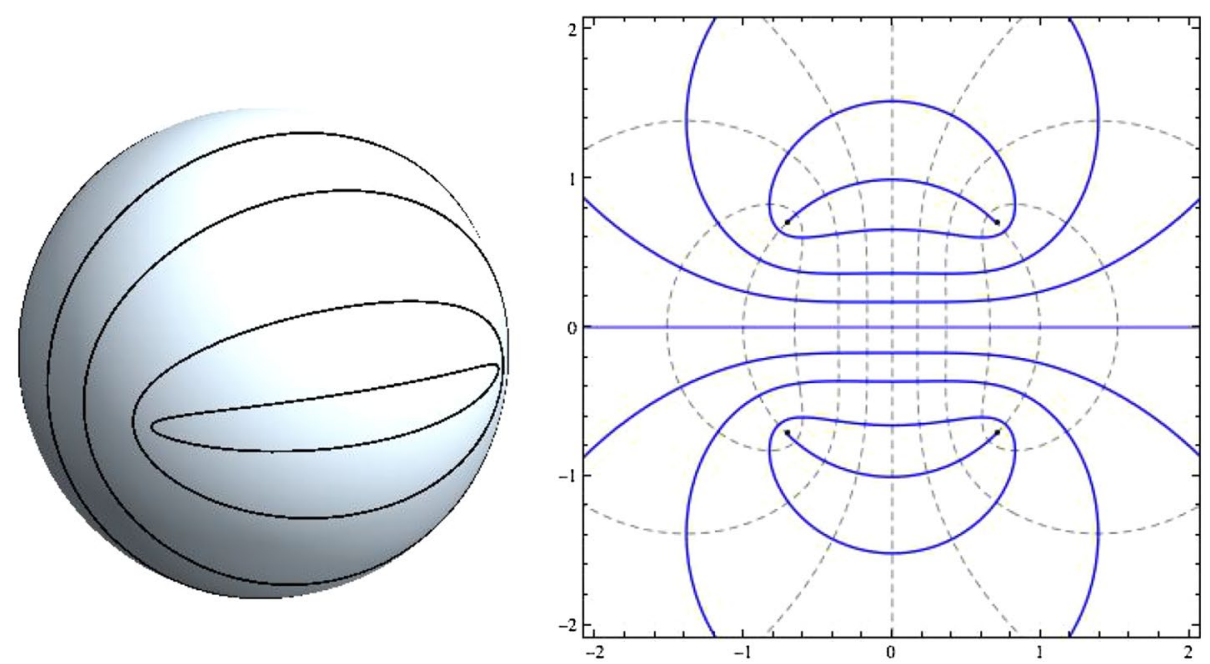

Fig. 5 Confocal family of ellipses in $S^{2}$ and in $\hat{\mathbb{C}}$

\section{Confocal ellipses in the sphere}

With a minor exception (Example 7.1), mirror foliations in $S^{2}$ are directly analogous to confocal ellipses in $E^{2}$. Figure 5 shows such a confocal family of ellipses on the sphere and the corresponding foliation obtained by stereographic projection to $\hat{\mathbb{C}}$. We observe: (1) There are actually two focal pairs; (2) The orthogonal hyperbolas (dashed curves, right) are actually ellipses!; (3) The foliation contains a geodesic and two geodesic arcs joining foci. These are easily explained, general features of confocal ellipses on the sphere.

The conformal model of the sphere is based on the usual identification of the unit sphere $S^{2}: X^{2}+Y^{2}+Z^{2}=1$ with $\hat{\mathbb{C}}$ via stereographic projection from the north pole $n=(0,0,1)$. Identifying $\mathbb{C}$ with the equatorial plane $Z=0(X+i Y \leftrightarrow(X, Y, 0))$, a point $p=(X, Y, Z) \in S^{2} \backslash\{n\}$ projects to $z=x+i y=\pi(p)=\frac{X+i Y}{1-Z}$ (the intersection of the line $\overline{n p}$ with the plane). Then, $\pi(n)=\infty$ completes the definition of the one-toone map $\pi: S^{2} \rightarrow \hat{\mathbb{C}}$.

It is well known that $\pi$ is conformal (angle-preserving) and maps circles to circles (circles through the north pole map to straight lines), and 'preserves' equatorial points $(\pi(X, Y, 0)=X+i Y)$. Since great circles meet the equator at antipodal points $\pm(X, Y, 0)$, geodesics are represented in $\hat{\mathbb{C}}$ by circles (or lines) which meet the unit circle $S^{1}:|z|=1$ at opposite points $\pm z$.

In general, a geodesic passing through the point $z$ passes through its antipode $-1 / \bar{z}$; conversely, any circle containing such an antipodal pair is a geodesic. Any two geodesics intersect in an antipodal pair $\left\{z_{0},-1 / \bar{z}_{0}\right\}$. Let $\omega_{z_{0}}$ denote the differential defining the elliptic pencil of oriented geodesics from $\mathrm{z}_{0}$ to $-1 / \bar{z}_{0}$; note that $\omega_{-1 / \bar{z}_{0}}=-\omega_{z_{0}}$. We have thus accounted for all oriented line pencils: $\left\{\mathcal{F}_{\omega_{z_{0}}}\right\}_{z_{0} \in S^{2}} \simeq S^{2}$.

Example 7.1 (Circular mirrors in $S^{2}$ ) The meridian lines from south pole to north pole are defined by $\omega_{0}=\mathrm{d} z / z>0$. Such "light rays" $\mathcal{F}_{\omega_{0}}$ on $S^{2}$ are reversed by the circular mirrors given by parallels of latitude: $Q=\omega_{0} \omega_{\infty}=-\mathrm{d} z^{2} / z^{2}$. 
Here, we are using a very special case of the following principle: since $\pi$ is angle preserving, the rule "angle of incidence equals angle of reflection" for geodesics on $S^{2}$ implies that the corresponding foliation on $\hat{\mathbb{C}}$ is the mirror foliation given by Definition 6.4.

Now any ordered pair of non-antipodal (and distinct) points $\left\{c_{1}, c_{2}\right\}$ in $\widehat{\mathbb{C}}$ may be chosen as foci (source and sink) for a confocal family of spherical ellipses, namely the mirror foliation $\mathcal{F}\left(c_{1}, c_{2}\right)=\mathcal{F}_{Q}$ defined by $Q=\omega_{1} \omega_{2}=\omega_{c_{1}}\left(-\omega_{c_{2}}\right)=\omega_{c_{1}} \omega_{-1 / \bar{c}_{2}}$. Note that the orthogonal foliation (by "hyperbolas") is defined by $-Q=\omega_{c_{1}} \omega_{c_{2}}$, but this also defines the confocal ellipses $\mathcal{F}\left(c_{1},-1 / \bar{c}_{2}\right)$, so we have verified property (2) above. (It is also easy to verify (3) at this point).

Remark 7.2 It is not hard to show that the above "optical" definition of a spherical ellipse is equivalent to the "string property": an ellipse in $S^{2}$ is the locus of points along which the spherical distances to a fixed pair of points sum to a constant $\left(d_{1}+d_{2}=L=\right.$ string length). The proof is not essentially harder than in the Euclidean case, but the advantage of being able to use conformal models of $S^{2}$ (rather than keeping track of spherical distances) is obviously much greater.

For explicit computations, it is convenient to "symmetrize" the four points $c_{1},-1 / \bar{c}_{1}, c_{2},-1 / \bar{c}_{2}$ using rotations of the sphere. Noting that these points lie on a geodesic, there are two natural ways to do this.

One way is to rotate so that the geodesic becomes the equator and the focal pairs are reflection symmetric w.r.t. the plane $Y=0$. Then, $c_{1}=c=e^{i \theta}, c_{2}=-\bar{c}$, where $0<\theta<\pi / 2$. Figure 5 shows the case $\theta=\pi / 4, c=\frac{1+i}{\sqrt{2}}$ (whose exceptional symmetry results in the orthogonal family $\mathcal{F}_{-Q}$ actually being congruent to $\mathcal{F}_{Q}$ !). The relevant differentials are: $\omega_{1}=\omega(c)=\frac{2 c \mathrm{~d} z}{z^{2}-c^{2}}, \quad \omega_{2}=-\omega(-\bar{c})=\omega(\bar{c})=\frac{2 \bar{c} \mathrm{~d} z}{z^{2}-\bar{c}^{2}}$ (using Propositions 3.2, 4.2), so $Q=\omega_{1} \omega_{2}=\frac{4 \mathrm{~d} z^{2}}{\left(z^{2}-c^{2}\right)\left(z^{2}-\bar{c}^{2}\right)}$.

An alternative is to rotate the sphere so that the four foci are real and a pair of these may be assumed to satisfy $c_{1}=1 / c, \quad c_{2}=-1 / c$, for some $c>1$. In Fig. $6, c=2$, so $c_{1}=\frac{1}{2}, c_{2}=\frac{-1}{2}$. The orthogonal "hyperbolas", (ellipses with foci at $\frac{1}{2}$ and 2 ) are also shown. In this view, it is tempting to interpret a hyperbola as a pair of curves (like the two branches in the Euclidean case); when the ellipses are described by quartic equations (in Part II), they will be seen also to come in pairs!

This time, $\omega_{1}=\omega\left(c_{1}\right)=\frac{\left(1+c^{2}\right) \mathrm{d} z}{(c z-1)(z+c)}$ and $\omega_{2}=-\omega\left(c_{2}\right)=\frac{\left(1+c^{2}\right) \mathrm{d} z}{(c z+1)(z-c)}$, so the geometric mean $\mathcal{F}_{Q}$ is defined by

$$
Q=\omega_{1} \omega_{2}=\frac{\left(1+c^{2}\right)^{2} \mathrm{~d} z^{2}}{\left(z^{2}-c^{2}\right)\left(c^{2} z^{2}-1\right)} .
$$

Thus, the natural parameter is the elliptic integral of the first kind

$$
\zeta=\Phi(z)=\int \sqrt{Q}=\int \frac{\left(1+c^{2}\right) \mathrm{d} z}{\sqrt{\left(z^{2}-c^{2}\right)\left(c^{2} z^{2}-1\right)}} .
$$

Its inverse $z=\phi(\zeta)$ is given by

$$
z=x+i y=\phi(\zeta)=\frac{1}{c} \operatorname{sn}\left(\frac{c^{2} \zeta}{c^{2}+1}, \frac{1}{c^{2}}\right)
$$




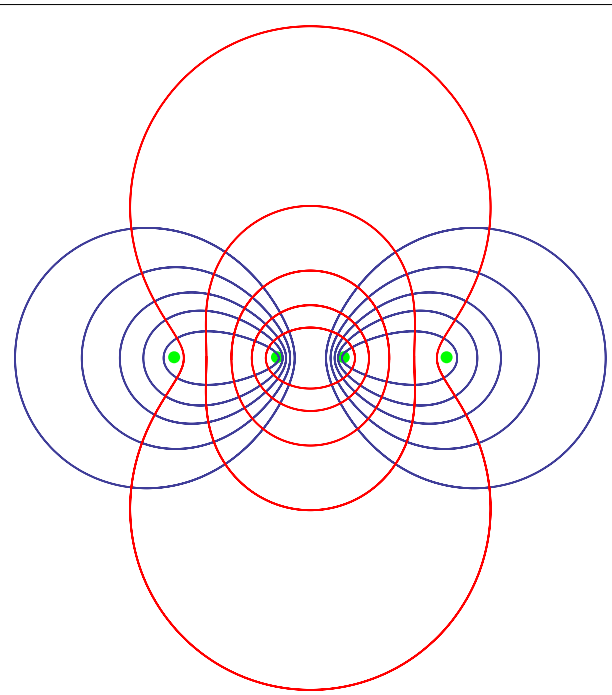

Fig. 6 Spherical ellipses (red) and hyperbolas (blue)

where sn $r$ is the Jacobi elliptic sine function (with modulus $k=\frac{1}{c^{2}}$ ).

Moreover, for fixed $s_{0} \in \mathbb{R}$, the horizontal trajectory $t \mapsto z=x+i y=\phi\left(t+i s_{0}\right)$ has real and imaginary parts $x(t), y(t)$ which may also be expressed in terms of $\operatorname{sn}(r ; k)$ and associated elliptic functions $\mathrm{cn} r=\sqrt{1-\mathrm{sn}^{2} r}, \quad \operatorname{dn} r=\sqrt{1-k^{2} \mathrm{sn}^{2} r}$. To be precise, let $u+i v_{0}=\frac{c^{2}\left(t+i s_{0}\right)}{c^{2}+1}$, let $k^{\prime}=\sqrt{1-k^{2}}=\sqrt{c^{4}-1} / c^{2}$ (the 'complementary modulus'), and compute the numbers $D=\operatorname{dn}\left(v_{0}, k^{\prime}\right), S=\operatorname{sn}\left(v_{0}, k^{\prime}\right), C=\operatorname{cn}\left(v_{0}, k^{\prime}\right)$. Then, the trajectory is given by

$$
x(t)=\frac{D \operatorname{sn}(u)}{c\left(1-S^{2} \operatorname{dn}^{2}(u)\right)} \quad y(t)=\frac{S C \operatorname{cn}(u) \mathrm{d} n(u)}{c\left(1-S^{2} \operatorname{dn}^{2}(u)\right)}
$$

In other words, we parameterize elliptic ellipses by elliptic functions!

\section{Concluding remarks}

Because of the familiarity of Euclidean conics and the very limited variety of spherical conics, the description of mirror foliations in $E^{2}$ and $S^{2}$ occupied only a minor portion of this introduction to the geometric mean. The corresponding discussion of mirror foliations in the hyperbolic case is complicated by the occurrence of three types of line pencils in $H^{2}$ and also more possibilities for coincidence of singularities between pencils of a pencil pair.

Even so, the analysis of mirror foliations in $H^{2}$ would seem to be a well-defined mathematical task, based on the description of line pencils and isometries in $H^{2}$ using, say, the upper half plane model or Poincare disk model (or preferably, both).

But there are also important questions of interpretation. For instance, should (nontrivial) mirror foliations in $H^{2}$ also count as confocal families of conics (as was the case in $E^{2}$ and $\left.S^{2}\right)$ ? For that matter, what is a focus in this context? How else to identify hyperbolic line pencils in $H^{2}$, geometrically, among geodesic foliations of $H^{2}$ ? Finally, what's the relationship between (leaves of) mirror foliations and the classical notion of nonEuclidean conic $([1,10])$ ? 
In fact, one cannot address all these questions satisfactorily without recourse to three or four different models of the hyperbolic plane. This is not the time for detailed explanations, but a vivid picture may be drawn in a few short paragraphs. (See, e.g., [7, 9] for background and closely related constructions).

Let $L \subset \mathbb{R}^{3}$ be an oriented line-the axis of the family of half-planes containing $L$. By intersecting these half-planes with the unit sphere $S^{2} \subset \mathbb{R}^{3}, L$ determines a foliation of $S^{2}$ by oriented circle arcs. It is in fact one of our oriented circle pencils in $\hat{\mathbb{C}} \simeq S^{2}$; conversely, all such pencils arise in this way (but the hyperbolic foliations by parallel circles correspond to parallel planes).

In particular, the oriented line pencils in spherical, Euclidean, and hyperbolic geometry are realized as follows. The (necessarily elliptic) pencils of lines in $S^{2}$ are precisely the cases for which $L$ meets $S^{2}$ in a pair of antipodal points. The elliptic pencils of lines in $E^{2} \simeq \mathbb{C}$ are precisely the cases for which $L$ meets $S^{2}$ at the north pole $n$ and one other point; parabolic pencils of lines in $E^{2}$ come from the axes $L$ which are tangent to $S^{2}$ at $n$.

Similarly, $H^{2}$ can be modeled as the (open) upper hemisphere $S_{+}^{2} \subset S^{2}$. Further, as it turns out: vertical axes $L$ which meet the equatorial plane inside, on, or outside the equator determine, respectively, elliptic, parabolic, and hyperbolic pencils of lines in $H^{2} \simeq S_{+}^{2}$.

Yet another useful model of $H^{2}$-the projective disk model $U$-may be obtained by vertical projection of $S_{+}^{2}$ onto the unit disk $U$ in the equatorial plane $\left(\simeq E^{2}\right)$. Geodesics in $U$ are now represented by lines in $E^{2}$ (projections of vertical half-planes) which intersect $U$. Further: a pencil of lines in $U$ is represented by a pencil of lines in $E^{2}$.

Such a pencil in $U$ is elliptic, parabolic, or hyperbolic, depending on whether lines of the pencil in $E^{2}$ meet inside, on, or outside the ideal boundary $S^{1}=\partial U$; the latter case includes parabolic pencils in $E^{2}$-the lines meet at an ideal point in the projective plane $\mathbb{R} P^{2}$. (In [9], such pencils are called interior, boundary, or exterior, but parabolic pencils in $E^{2}$ are not mentioned). To sum up: lines of a pencil in $U$ may meet at a finite point of $U$, or at infinity (at an ideal point of $U$ ), or beyond infinity, or beyond the beyond!

Authors' contributions

Both authors read and approved the final manuscript.

Acknowledgements

Not applicable.

Competing interests

The authors declare that they have no competing interests.

\section{Publisher's Note}

Springer Nature remains neutral with regard to jurisdictional claims in published maps and institutional affiliations.

Received: 8 August 2018 Accepted: 3 October 2018

Published online: 08 November 2018

\section{References}

1. Coolidge, J.L.: The Elements of Non-Euclidean Geometry. Oxford at the Clarendon Press, Oxford (1909)

2. Langer, J.C.: On meromorphic parameterizations of real algebraic curves. J. Geom. 100(1-2), 105-128 (2011)

3. Langer, J.C.: Plotting the polyhedral geometry of a quadratic differential. J. Geom. (2017). https://doi.org/10.1007/ s00022-017-0378-y

4. Langer, J.C., Singer, D.A.: Foci and foliations of algebraic curves. Milan J. Math. 75, 225-271 (2007)

5. Langer, J.C., Singer, D.A.: Flat curves. Mediterr. J. Math. 14, 236 (2017) 
6. Muciño-Raymundo, J.: Complex structures adapted to smooth vector fields. Math. Ann. 322(2), 229-265 (2002)

7. Schwerdtfeger, H.: Geometry of Complex Numbers. Dover Publications, New York (1979)

8. Singer, D.A.: The location of critical points of finite Blaschke products. Conform. Geom. Dyn. 10, 117-124 (2006)

9. Stillwell, J.: Geometry of Surfaces. Springer, New York (1992)

10. Story, W.: On the non-Euclidean properties of conics. Am. J. Math. 5(1), 358-381 (1882)

11. Strebel, K.: Quadratic Differentials. Ergebnisse der Mathematik und ihrer Grenzgebeite, vol. 5. Springer, Berlin (1984)

Submit your manuscript to a SpringerOpen ${ }^{0}$ journal and benefit from:

- Convenient online submission

- Rigorous peer review

Open access: articles freely available online

- High visibility within the field

- Retaining the copyright to your article

Submit your next manuscript at $\gg$ springeropen.com 